TRANSACTIONS OF THE

AMERICAN MATHEMATICAL SOCIETY

Volume 311, Number 1, January 1989

\title{
PRIMARY CYCLES ON THE CIRCLE
}

\author{
LOUIS BLOCK, ETHAN M. COVEN, LEO JONKER AND MICHAL MISIUREWICZ
}

\begin{abstract}
We consider cycles, i.e., periodic orbits, of continuous degree one maps of the circle. One cycle forces another if every such map that has a cycle which looks like the first also has a cycle which looks like the second. We characterize primary cycles, i.e., those which force no other cycle of the same period.
\end{abstract}

\section{INTRODUCTION}

This paper concerns cycles, i.e., periodic orbits, of continuous degree one maps of the circle. As is customary in this subject, we deal with lifts of maps and cycles to the reals rather than with maps and cycles themselves.

We say that $P \subseteq \mathbf{R}$ is a cycle if it has the properties of a lift of a cycle of a continuous degree one map of the circle, and that $P$ forces $Q$ if every map that has a cycle which looks like $P$ also has one which looks like $Q$. (See $\S 2$ for precise definitions.) "Looks like" is really an equivalence relation on cycles, and the proper formal setting for forcing is equivalence classes of cycles, rather than cycles themselves. However, most of the proofs use actual cycles, so we sacrifice the precision of equivalence classes in order to avoid an additional layer of notation.

We show that "forces" is a partial order on (equivalence classes of) cycles and that each cycle forces only finitely many inequivalent cycles of a given period. Thus for each $n>0$, there are cycles of period $n$ which force no inequivalent cycle of period $n$. We call such cycles primary. The main results of this paper are Theorem 4.1, which characterizes primary cycles, and Theorem 3.15, which characterizes cycles having a trivial rotation interval.

Forcing for maps of the interval has been studied by a number of authors, most systematically by S. Baldwin [Ba]. L. Alsedà, J. Llibre and R. Serra [ALS], L. Block and W. A. Coppel [BC], Coppel [C], and C.-W. Ho $[\mathrm{H}]$ all deal with the problem of characterizing primary interval cycles. We summarize their results in $\S 1$. Forcing, with emphasis on primary cycles, for maps of the interval and of the space $Y=\left\{z \in \mathbf{C}: 0 \leq z^{3} \leq 1\right\}$ has been studied by Alsedà, Llibre and M. Misiurewicz [ALM]. Special cases of forcing for maps of the circle have been considered by Alsedà, Llibre, Misiurewicz and C. Simó [ALMS], A. Chenciner,

Received by the editors October $15,1987$.

1980 Mathematics Subject Classification (1985 Revision). Primary 58F20.

(c) 1989 American Mathematical Society $0002-9947 / 89 \$ 1.00+\$ .25$ per page 
J.-M. Gambaudo and Ch. Tresser [CGT], Llibre [L], R. MacKay and Tresser [MT], Misiurewicz [M2], and S. Newhouse, J. Palis and F. Takens [NPT].

This paper was made possible by invitations to various subsets of the authors from Queen's University, the University of Warwick, and the Stefan Banach International Mathematical Center.

\section{BACKGROUND FROM THE INTERVAL}

Definitions. (1) A(n interval) cycle of period $n$ is a cyclic permutation $\alpha: X \rightarrow$ $X=\left\{x_{1}<\cdots<x_{n}\right\} \subseteq \mathbf{R}$ of an $n$-element set of real numbers. We often suppress $\alpha$ and refer to $X$ as the cycle. The period of $X$ is denoted by $|X|$.

(2) Cycles $\alpha: X \rightarrow X$ and $\beta: Y \rightarrow Y$ are equivalent if $|X|=|Y|$, and $\alpha\left(x_{i}\right)=x_{j}$ if and only if $\beta\left(y_{i}\right)=\left(y_{j}\right)$.

(3) An interval map is a continuous map $f: I \rightarrow I$ from a compact interval to itself.

(4) An $f$-cycle is a set $X \subseteq I$ such that $\left.f\right|_{X}: X \rightarrow X$ is a cycle.

(5) $X$ forces $Y$ if every interval map which has a cycle equivalent to $X$ also has one equivalent to $Y$.

Baldwin [Ba, Theorem 1.4] proved that "forces" is a partial order on the set of equivalence classes of cycles. See also [ALM].

Definition. A cycle $X$ is primary if every cycle of period $|X|$ which is forced by $X$ is equivalent to $X$.

Since there are only finitely many equivalence classes of a given period, it follows that every cycle forces a primary cycle of the same period.

Theorem 1.1 below characterizes primary cycles.

Definitions. (1) A cycle $\alpha: X \rightarrow X$ of period $n$ has a block structure over a cycle $\beta: Y \rightarrow Y=\left\{y_{1}<\cdots<y_{m}\right\}$ of period $m$ if $n$ is a multiple of $m$, say $n=s m$, and if $X$ is written $X=X_{1} \cup \cdots \cup X_{m}$, where $X_{i}=\left\{x_{(i-1) s+1}<\cdots<\right.$ $\left.x_{(i-1) s+s}\right\}$, then $\alpha\left(X_{i}\right)=X_{j}$ if and only if $\beta\left(y_{i}\right)=y_{j}$.

(2) $X$ is a $Z$-extension of $Y$ if it has a block structure over $Y$ as in (1), such that for some $k, 1 \leq k \leq m, \alpha$ is monotone on $X_{i}$ for all $i \neq k$, and $\left.\alpha^{m}\right|_{X_{k}}: X_{k} \rightarrow X_{k}$ is equivalent to $Z$.

(3) A 2-extension is one in which $Z$ is a two-point cycle. (There is just one equivalence class of two-point cycles.)

(4) A Štefan cycle is a cycle $\alpha: X \rightarrow X$ of odd period $n \geq 3$, such that $X$ can be written $\left\{\bar{x}_{n}<\bar{x}_{n-2}<\cdots<\bar{x}_{3}<\bar{x}_{1}<\bar{x}_{2}<\cdots<\bar{x}_{n-1}\right\}$, or with the inequalities reversed, and $\alpha\left(\bar{x}_{1}\right)=\bar{x}_{2}, \ldots, \alpha\left(\bar{x}_{n-1}\right)=\bar{x}_{n}$, and $\alpha\left(\bar{x}_{n}\right)=\bar{x}_{1}$. (For each odd $n \geq 3$, there are exactly two equivalence classes of Štefan cycles.)

(5) A Stefan extension is one in which $Z$ is a Štefan cycle.

Theorem 1.1 (cf. [ALM, Remark 9.2]). The following statements about a cycle $X$ are equivalent.

(1) $X$ is primary. 
(2) $X$ can be obtained from the one-point cycle by a finite number (possibly zero) of 2-extensions and then at most one Sttefan extension.

(3) There is an interval map whose only cycle of period $|X|$ is $X$.

Proof. (1) $\Leftrightarrow(2)$ is implicit in [ک̌ ] for $|X|$ odd and in [Bl] for $|X|$ a power of two. For $|X|$ even and not a power of two, (1) $\Leftrightarrow(2)$ was conjectured by Baldwin [Ba, Conjecture 6.3], and (1) $\Rightarrow(2)$ was proved by Block and Coppel [BC, Theorem 1].

Clearly $(3) \Rightarrow(1)$. We show that (2) $\Rightarrow(3)$. Let $\alpha: X \rightarrow X=\left\{x_{1}<\cdots<x_{n}\right\}$. If $n=1$, let $f$ be the constant map $f(x)=x_{1}$, defined on any nondegenerate compact interval containing $x_{1}$. If $n \geq 2$ is a power of two, let $f:\left[x_{1}, x_{n}\right] \rightarrow$ $\left[x_{1}, x_{n}\right]$ agree with $\alpha$ on $X$ and be monotone quadratic on $\left[x_{1}, x_{2}\right]$ and linear on $\left[x_{i}, x_{i+1}\right]$ for $i \neq 1$. If $n$ is not a power of two, let $f:\left[x_{1}, x_{n}\right] \rightarrow\left[x_{1}, x_{n}\right]$ agree with $\alpha$ on $X$ and be linear on each $\left[x_{i}, x_{i+1}\right]$. In each case, it is easy to check, using the Markov graph, that $X$ is the only $f$-cycle of period $n$.

There is no standard terminology in this area. Cycles satisfying (1) are called minimal in [Ba] and primary in this paper, those satisfying (2) are called strongly simple in [BC], and those satisfying (3) are called primary in [ALM]. Cycles are often called (periodic) orbits.

\section{CYCLES AND FORCING ON THE CIRCLE}

Let $f$ be a continuous degree one map of the circle, and let $C$ be an $f$-cycle of period $n$. Let $\pi: \mathbf{R} \rightarrow \mathbf{R} / \mathbf{Z}$ be the canonical projection onto the circle and let $F$ be a lift of $f$. Then, writing $\pi^{-1}(C)=\left\{\cdots<x_{0}<x_{1}<\cdots\right\}$, we have

(a) $x_{i+n}=x_{i}+1$.

(b) For all $x \in \mathbf{R}, F(x+1)=F(x)+1$.

(c) For all $x, y \in \pi^{-1}(C)$, there are integers $m>0$ and $s$ such that $F^{m}(x)=y+s$.

(d) There is an integer $r$ such that for all $x \in \pi^{-1}(C), F^{n}(x)=x+r$.

(d) follows from (a), (b), and (c). See Lemma 2.1 below.

We abstract this situation, keeping only $\left.F\right|_{\pi^{-1}(C)}$.

Definition. A (degree one) cycle of period $n$ is a map $\varphi: P \rightarrow P=\left\{\cdots<x_{0}<\right.$ $\left.x_{1}<\cdots\right\} \subseteq \mathbf{R}$ such that

(a) $x_{i+n}=x_{i}+1$.

(b) For all $x \in P, \varphi(x+1)=\varphi(x)+1$.

(c) For all $x, y \in P$, there are integers $m>0$ and $s$ such that $\varphi^{m}(x)=$ $y+s$.

We often suppress $\varphi$ and refer to $P$ as the cycle. The period of $P$ is denoted by $|P|$.

Lemma 2.1. Let $\varphi: P \rightarrow P$ be a cycle of period $n$. There is an integer $r$ such that for all $x \in P, \varphi^{n}(x)=x+r$. 
Proof. Let $\tilde{\varphi}(x)=\varphi(x)-[\varphi(x)]$, i.e., the fractional part of $\varphi(x)$. Then $\tilde{\varphi}$ is a cyclic permutation of the $n$-element set $P \cap[0,1)$. Therefore $\tilde{\varphi}^{n}(x)=x$ for all $x \in P \cap[0,1)$, and so for each $x \in P$, there is an integer $r$ such that $\varphi^{n}(x)=x+r$. By (b) and (c), $r$ does not depend on $x$.

Definitions. (1) The number $\rho(P)=r / n$ of Lemma 2.1 is called the rotation number of $P$. (Writing $\rho(P)=r / n$ does not imply that $\operatorname{GCD}(r, n)=1$.)

(2) Cycles $\varphi: P \rightarrow P$ and $\psi: Q \rightarrow Q$ are equivalent if $|P|=|Q|$ and we can write $P=\left\{\cdots<x_{0}<x_{1}<\cdots\right\}$ and $Q=\left\{\cdots<y_{0}<y_{1}<\cdots\right\}$ in such a way that $\varphi\left(x_{i}\right)=x_{j}$ if and only if $\psi\left(y_{i}\right)=y_{j}$.

(3) Let $\mathscr{C}$ denote the class of continuous maps $F: \mathbf{R} \rightarrow \mathbf{R}$ which are lifts of continuous degree one maps of the circle; i.e., $F \in \mathscr{C}$ if and only if $F$ is continuous and $F(x+1)=F(x)+1$ for all $x \in \mathbf{R}$. Thus $\mathscr{C}$ is the class of continuous old maps. ("Old" is an anagram of an acronym: "lift of degree one" [M3]. We pass up the chance to call the maps in $\mathscr{C}$ "cold.")

(4) Let $F \in \mathscr{C}$. An $F$-cycle is a set $P \subseteq \mathbf{R}$ such that $\left.F\right|_{P}: P \rightarrow P$ is a cycle.

(5) $P$ forces $Q$ if every map in $\mathscr{C}$ which has a cycle equivalent to $P$ also has a cycle equivalent to $Q$.

Note that $\varphi$ and $\varphi+1$ are not equivalent, although they represent the same cycle on the circle. Making $\varphi$ and $\varphi+1$ equivalent would negate the benefit of dealing with lifted cycles rather than cycles on the circle itself and would not allow meaningful comparison of rotation numbers of different cycles.

The main result of this section is Theorem 2.6 , which states that "forces" is a partial order on equivalence classes of cycles.

Definition. Let $F \in \mathscr{C}$ and let $P$ be an $F$-cycle. $F$ is $P$-monotone if it is (not necessarily strictly) monotone between adjacent members of $P$.

Lemma 2.2. Let $F$ be $P$-monotone. Then $P$ forces $Q$ if and only if $F$ has a cycle equivalent to $Q$.

Proof. Whether $P$ forces $Q$ is determined by the oriented Markov graph of $P$ and the equivalence classes of $P$ and $Q$. (Cf. [Ba, Theorem 3.3], [Be, Lemma 1.8], or [ALM, Lemma 1.18], the corresponding result for interval cycles.)

Lemma 2.3. Let $F \in \mathscr{C}$. If $F$ is piecewise polynomial and each piece is of degree at least two, then $F$ has only finitely many cycles of each period.

Proof. Let $n>0$ be given. Since $\left\{F^{n}(x)-x: x \in[0,1]\right\}$ is compact, there are only finitely many integers $r$ such that $F^{n}(x)=x+r$ has a solution in $[0,1]$. Fix such an $r$. Since $F^{n}-i d$ is piecewise polynomial, and none of its pieces is constant, there are only finitely many $x \in[0,1]$ which satisfy $F^{n}(x)=x+r$.

Lemma 2.4. For every cycle $P$, there is a $P$-monotone map $F$ which has only finitely many cycles of each period. 
Proof. Let $\varphi: P \rightarrow P$. Let $F$ agree with $\varphi$ on $P$, be monotone quadratic on each $\left[x_{i}, x_{i+1}\right]$, and satisfy $F(x+1)=F(x)+1$ for all $x \in \mathbf{R}$. The result now follows from Lemma 2.3.

Definition. Let $F \in \mathscr{C}$, let $n>0$ be an integer, and let $Q=\left\{\cdots<y_{0}<y_{1}<\right.$ $\cdots\}$ be a set which satisfies $y_{i+n}=y_{i}+1$ for each $i$. The $F$-variation of $Q$ is $\operatorname{var}_{F}(Q)=\sum_{1 \leq i \leq n}\left|F\left(y_{i}\right)-F\left(y_{i-1}\right)\right|$. (This number is well defined; i.e., it does not change if $\bar{Q}$ is renumbered.)

Lemma 2.5. Let $F \in \mathscr{C}$ and let $P$ be an $F$-cycle. If $P$ forces $Q$ but $P$ and $Q$ are not equivalent, then $F$ has a cycle $Q^{\prime}$, equivalent to $Q$, such that $\operatorname{var}_{F}\left(Q^{\prime}\right)<\operatorname{var}_{F}(P)$.

Proof. Let $P=\left\{\cdots<x_{0}<x_{1}<\cdots\right\}$. Then $\left.F\right|_{P}=\varphi$ is not increasing. For otherwise, if $\varphi^{n}(x)=x+r$ for all $x \in P$, then $\varphi$ is equivalent to $\psi: \mathbf{Z} / n \rightarrow$ $\mathbf{Z} / n=\{i / n: i \in \mathbf{Z}\}$, defined by $\psi(y)=y+r / n$, and hence by Lemma 2.2, $P$ forces only cycles equivalent to itself.

Let $G \in \mathscr{C}$ be $P$-monotone. Since $P$ forces $Q, G$ has a cycle $R=\{\cdots<$ $\left.z_{0}<z_{1}<\cdots\right\}$ equivalent to $Q$. As in the proofs of [Ba, Theorem 3.3], [Be, Lemma 1.8], and [ALM, Lemma 1.18], $F$ has a cycle $Q^{\prime}=\left\{\cdots<y_{0}<y_{1}<\right.$ $\cdots\}$, equivalent to $Q$, labeled in such a way that for all $i, j, k, x_{i}<y_{j}<x_{i+1}$ if and only if $x_{i}<z_{j}<x_{i+1}$, and $F\left(y_{j}\right)=y_{k}$ if and only if $G\left(z_{j}\right)=z_{k}$. It follows that if $s \geq 0$ and $x_{i}<y_{j}<y_{j+1}<\cdots<y_{j+s}<x_{i+1}$, then either $F\left(x_{i}\right)<F\left(y_{j}\right)<\cdots<F\left(y_{j+s}\right)<F\left(x_{i+1}\right)$ or $F\left(x_{i}\right)>F\left(y_{j}\right)>\cdots>$ $F\left(y_{j+s}\right)>F\left(x_{i+1}\right)$, because either $G\left(x_{i}\right)<G\left(z_{j}\right)<\cdots<G\left(z_{j+s}\right)<G\left(x_{i+1}\right)$ or $G\left(x_{i}\right)>G\left(z_{j}\right)>\cdots>G\left(z_{j+s}\right)>G\left(x_{i+1}\right)$. Thus $\operatorname{var}_{F}(P)=\operatorname{var}_{F}\left(P \cup Q^{\prime}\right)$. On the other hand, since $\left.F\right|_{P}$ is not monotone, $\operatorname{var}_{F}\left(Q^{\prime}\right)<\operatorname{var}_{F}\left(P \cup Q^{\prime}\right)$.

Theorem 2.6. "Forces" is a partial order on equivalence classes of cycles.

Proof. Since "forces" is reflexive and transitive on equivalence classes, it suffices to show that it is antisymmetric; i.e., if $P$ forces $Q$ and $Q$ forces $P$, then $P$ is equivalent to $Q$.

Suppose that there are cycles $P$ and $Q$ such that $P$ forces $Q$ and $Q$ forces $P$, but $P$ is not equivalent to $Q$. By Lemma 2.4, there is a map $F \in \mathscr{C}$ for which $P$ is an $F$-cycle and which has only finitely many cycles of each period. Applying Lemma 2.5 to $F$ inductively, alternating the statements " $P$ forces $Q$ " and " $Q$ forces $P$," we find that $F$ has cycles $P=P_{0}, P_{1}, P_{2}, \ldots$, all equivalent to $P$, and cycles $Q_{1}, Q_{2}, \ldots$, all equivalent to $Q$, such that for each $i \geq 0, \operatorname{var}_{F}\left(P_{i}\right)>\operatorname{var}_{F}\left(Q_{i+1}\right)>\operatorname{var}_{F}\left(P_{i+1}\right)$. This violates the choice of $F$.

\section{TWIST AND PRIMARY CYCLES}

Definition. A cycle $\varphi: P \rightarrow P$ is a twist cycle if $\varphi$ is increasing.

The term "twist" is justified by the following fact, noted in the proof of Lemma 2.5. A twist cycle with period $n$ and rotation number $r / n$ is equivalent 
to the cycle $\psi: \mathbf{Z} / n \rightarrow \mathbf{Z} / n$, defined by $\psi(x)=x+r / n$. If $P$ is a twist cycle of period $n$ and rotation number $r / n$, then $\operatorname{GCD}(r, n)=1$. In particular, there is only one equivalence class of twist cycles with a given rotation number.

Theorem 3.1. Every cycle forces a twist cycle with the same rotation number.

Proof. Let $P$ be a cycle and let $F$ be $P$-monotone. By [ALMS, Theorem A] (or [M2, Theorem B] or [CGT, Theorem 2]), $F$ has a twist cycle with the same rotation number as $P$.

Theorem 3.2 shows that twist cycles may be thought of as "prime."

Theorem 3.2. The following statements about a cycle $P$ are equivalent.

(1) $P$ is a twist cycle.

(2) Every cycle with rotation number $\rho(P)$ which is forced by $P$ is equivalent to $P$.

(3) Every cycle which is forced by $P$ is equivalent to $P$.

(4) There is a map in $\mathscr{C}$ whose only cycle is $P$.

Proof. (4) $\Rightarrow(3)$ and (3) $\Rightarrow(2)$ are obvious, and (2) $\Rightarrow(1)$ follows from Theorem 3.1. To prove $(1) \Rightarrow(4)$, let $\varphi: P \rightarrow P=\left\{\cdots<x_{0}<x_{1}<\cdots\right\}$ have rotation number $r / n$ (in lowest terms). Then, as noted after the definition of twist cycle, $\varphi\left(x_{i}\right)=x_{i+r}$ for all $i$. Let $F$ agree with $\varphi$ on $P$ and be monotone quadratic on $\left[x_{i}, x_{i+1}\right]$ if $i \equiv 0(\bmod n)$ and linear on $\left[x_{i}, x_{i+1}\right]$ if $i \not \equiv 0(\bmod n)$.

Definition. A cycle $P$ is primary if every cycle of period $|P|$ which is forced by $P$ is equivalent to $P$.

Theorem 3.3. $P$ is primary if every cycle of period $|P|$ and rotation number $\rho(P)$ which is forced by $P$ is equivalent to $P$.

Before proving Theorem 3.3, we derive a corollary.

Corollary 3.4. Every cycle forces a primary cycle with the same period and rotation number.

Proof. Let $P$ be a cycle and let $\mathscr{P}$ denote the set of cycles forced by $P$ and having the same period and rotation number as $P$. Then $\mathscr{P} \neq \varnothing(P \in \mathscr{P})$ and by Lemma $2.4, \mathscr{P}$ is finite. Hence by Theorem 2.6 , there is a cycle in $\mathscr{P}$ which is equivalent to any cycle in $\mathscr{P}$ that it forces. This cycle must be primary.

We will prove Theorem 3.3 by examining rotation intervals [NPT, Chapter III, §3].

Definitions. Let $F \in \mathscr{C}$ and let $x \in \mathbf{R}$. The number

$$
\rho(x)=\limsup _{n \rightarrow \infty}\left(F^{n}(x)-x\right) / n
$$

is called the rotation number of $x$. The closed interval $\left[\rho_{-}(F), \rho_{+}(F)\right]$, where $\rho_{-}(F)=\inf \{\rho(x): x \in \mathbf{R}\}$ and $\rho_{+}(F)=\sup \{\rho(x): x \in \mathbf{R}\}$ is called the 
rotation interval of $F$. When $\rho_{-}(F)=\rho_{+}(F)$, we denote this number by $\rho(F)$.

If a map in $\mathscr{C}$ is nondecreasing, then its rotation interval is trivial. The converse is false. (See the example near the end of this section.)

Lemma 3.5 [NPT, Chapter III, Corollary 3.2]. If $\rho_{-}(F) \leq r / n \leq \rho_{+}(F)$, then $F$ has a cycle with rotation number $r / n$.

Definition. For a cycle $P$, let $\rho_{-}(P)=\rho_{-}(F)$ and $\rho_{+}(P)=\rho_{+}(F)$, where $F$ is any $P$-monotone map. (These numbers are well defined.) The rotation interval of $P$ is $\left[\rho_{-}(P), \rho_{+}(P)\right]$.

We show in Theorem 3.14 that primary cycles have trivial rotation intervals. Theorem 3.15 characterizes cycles which have trivial rotation intervals.

Lemma 3.6. $\rho_{-}(P)=\inf \{\rho(Q): P$ forces $Q\}$ and $\rho_{+}(P)=\sup \{\rho(Q): P$ forces $Q\}$.

Proof. Apply Lemma 3.5.

Another way to obtain the rotation interval of $F$ is to introduce (as in [ALMS]) the maps $F_{-}$and $F_{+}$.

Definition. $F_{-}(x)=\inf _{y \geq x} F(y)$ and $F_{+}(x)=\sup _{y \leq x} F(y)$.

Note that $F_{-} \leq F \leq F_{+}, F_{-}, F_{+} \in \mathscr{C}$, and $F_{-}$and $F_{+}$are nondecreasing and hence have trivial rotation intervals. For each $n>0,\left(F_{-}\right)^{n}=\left(F^{n}\right)_{-}$and $\left(F_{+}\right)^{n}=\left(F^{n}\right)_{+}$. Hence the symbols $F_{-}^{n}$ and $F_{+}^{n}$ are unambiguous.

Lemma 3.7. $\rho_{-}(F)=\rho\left(F_{-}\right)$and $\rho_{+}(F)=\rho\left(F_{+}\right)$.

Proof. A routine exercise.

Definition. The nonconstant part of a map is the complement of the union of the open intervals on which the map is constant.

Lemma 3.8. Let $F$ be $P$-monotone and satisfy either $\rho(P)=\rho_{-}(P)$ or $\rho(P)=$ $\rho_{+}(P)$. Then $F$ has a twist cycle $Q$ such that

(1) $\rho(P)=\rho(Q)$.

(2) For $x \in P$ and $y \in Q, x<y$ if and only if $F(x)<F(y)$.

Proof. Let $\rho(P)=\rho_{+}(P)=r / n$, where $n=|P|$. By Lemma 3.5 (applied to $\left.F_{+}\right)$, there is a point $z \in \mathbf{R}$ such that $F_{+}^{n}(z)=z+r$. Since $F_{+}^{n} \in \mathscr{C}, z$ may be chosen to lie on the nonconstant part of $F_{+}^{n}$. Then $z, F_{+}(z), \ldots, F_{+}^{n-1}(z)$ all lie on the nonconstant part of $F_{+}$. Therefore $F=F_{+}$at these points. Thus there is an $F$-cycle $Q$ which contains $z$. Since $F=F_{+}$on $Q$ and $F_{+}$is nondecreasing, $Q$ is a twist cycle. Clearly $\rho(Q)=r / n$.

Now let $x \in P, y \in Q$, with $x \neq y$. Then $F(x) \neq F(y)$. If $x<y$, then (since $F \leq F_{+}, F_{+}$is nondecreasing, and $F_{+}=F$ on $Q$ ) we have $F(x) \leq$ $F_{+}(x) \leq F_{+}(y)=F(y)$. Conversely, suppose $x>y$ and $F(x)<F(y)$. Since $F^{n}(x)=x+r$ and $F^{n}(y)=y+r$, there is an integer $k, 0<k<n$, such that 
$F^{k}(x)<F^{k}(y)$ and $F^{k+1}(x)>F^{k+1}(y)$. But $F^{k}(x) \in P$ and $F^{k}(y) \in Q$, so this can't happen.

If $\rho(P)=\rho_{-}(P)=r / n$, the same argument works with $F_{-}$in place of $F_{+}$ and the inequalities reversed.

Lemma 3.9. Let $F$ be a $P$-monotone map which has a twist cycle $Q$ such that for $x \in P$ and $y \in Q, x<y$ if and only if $F(x)<F(y)$. Then $\rho_{-}(P)=\rho_{+}(P)$. Proof. If $P=Q$, there is nothing to prove. So we assume that $P \neq Q$ and hence $P \cap Q=\varnothing$. It suffices to show

(*) $\quad$ For $z \in \mathbf{R}$ and $y \in Q, y<z$ if and only if $F(y)<F(z)$,

for then $\rho_{-}(F)=\rho_{+}(F)=\rho(Q)$.

Let $y<y^{\prime}$ be consecutive points of $Q$. Because $F$ is $P$-monotone and $P \cap Q=\varnothing, F\left[y, y^{\prime}\right]$ is the convex hull of the finite set $\left\{F(y), F\left(y^{\prime}\right)\right\} \cup$ $\left\{F(x): x \in P \cap\left[y, y^{\prime}\right]\right\}$. But $F(y)<F(x)<F\left(y^{\prime}\right)$ for all $x \in P \cap\left[y, y^{\prime}\right]$. Hence $F\left[y, y^{\prime}\right]=\left[F(y), F\left(y^{\prime}\right)\right]$, and $(*)$ follows.

Lemmas 3.8 and 3.9 together yield a characterization of cycles with trivial rotation intervals. A more tractable characterization is given by Theorem 3.15 at the end of this section.

Lemma 3.10. For any cycle $P$, either $\rho_{-}(P)<\rho(P)<\rho_{+}(P)$ or $\rho_{-}(P)=$ $\rho(P)=\rho_{+}(P)$.

Proof. Apply Lemmas 3.8 and 3.9.

Lemma 3.11 (cf. [M1, Proposition 4; M2, Proposition 5.1]). If $\rho_{-}(F)<r / n<$ $\rho_{+}(F)$, then $F$ has a cycle with period $n$ and rotation number $r / n$ which lies on the nonconstant part of $F$.

Proof. Write $r / n=s / m$ where $\operatorname{GCD}(s, m)=1$, and set $G=F^{m}-s$. Then $\rho_{-}(G)<0<\rho_{+}(G)$, and hence by Lemma 3.5, $G$ has a cycle with a negative rotation number and one with a positive rotation number. Therefore, by [ALMS, Theorem A], $G$ has a twist cycle $P$ with a positive rotation number and a twist cycle $Q$ with a negative rotation number. Choose $x \in P$ and $y \in Q$ so that $x<y$ and $(x, y) \cap(P \cup Q)=\varnothing$. Then $G(y)<x<y<G(x)$. Let $I=[G(y), x]$ and $J=[y, G(x)]$. Then each of $I$ and $J \quad G$-covers both $I$ and $J$ in an orientation-preserving manner. (We say that $[a, b] G$-covers $[c, d]$ in an orientation-preserving manner if $G(a) \leq c$ and $G(b) \geq d$.)

Let $t=n / m$, and let $K_{0}=I, K_{1}=K_{2}=\cdots=K_{t-1}=J, K_{t}=I$. (If $t=$ 1 , let $K_{0}=K_{1}=I$.) Let $L_{t}=K_{t}$, and inductively construct subintervals $L_{i} \subseteq$ $K_{i}, t-1 \geq i \geq 0$, such that $L_{i} G$-covers $L_{i+1}$ in an orientation-preserving manner and $G\left(L_{i}\right)=L_{i+1}$. Then $z=\sup \left\{w \in L_{0}: G^{t}(w)=w\right\}$ has $G$-period $t$ and lies on the nonconstant part of $G^{t}$, and hence $z, G(z), \ldots, G^{t-1}(z)$ all lie on the nonconstant part of $G$.

The $F$-cycle which contains $z$ has rotation number $r / n$, and by [M1, Lemma 5] period $t m=n$. Since $z, G(z), \ldots, G^{t-1}(z)$ all lie on the nonconstant part 
of $G$, they lie on the nonconstant part of $F^{m}$, and hence $z, F(z), \ldots, F^{n-1}(z)$ all lie on the nonconstant part of $F$.

Lemma 3.12. Let $\rho_{-}(F) \leq a \leq b \leq \rho_{+}(F)$. Then there exists a map $G \in \mathscr{C}$ which agrees with $F$ on the nonconstant part of $G$, such that $\rho_{-}(G)=a$ and $\rho_{+}(G)=b$.

Proof. For $t \geq 0$, set $F_{t}(x)=\min \left\{F(x), F_{-}(x)+t\right\}$. Note that $F_{t} \in \mathscr{C}$. Since $F_{-}$agrees with $F$ on its nonconstant part, so does each $F_{t}$. Since the mappings $t \mapsto F_{t}$ and [NPT, Chapter III, Proposition 3.3] $G \mapsto \rho_{-}(G)$ and $G \mapsto \rho_{+}(G)$ are continuous (with spaces of maps having the $C^{0}$ topology), the mappings $t \mapsto \rho_{-}\left(F_{t}\right)$ and $t \mapsto \rho_{+}\left(F_{t}\right)$ are continuous. But $F_{0}=F_{-}$, and for $t$ large enough, $F_{t}=F$. Thus $\rho_{+}\left(F_{0}\right) \leq a$, and for $t$ large enough, $\rho_{+}\left(F_{t}\right) \geq b$. Therefore there exists $t_{0} \geq 0$ such that $\rho_{+}\left(F_{t_{0}}\right)=b$. But $\left(F_{t}\right)_{-}=F_{-}$for all $t \geq 0$, and so by Lemma 3.7, $\rho_{-}\left(F_{t_{0}}\right)=\rho_{-}(F)$.

Now make a similar construction, starting with $F_{t_{0}}$ instead of $F$, and with the subscripts + and - interchanged. We obtain a map $G$ which agrees with $F_{t_{0}}$, and hence with $F$, on its nonconstant part, such that $\rho_{-}(G)=a$ and $\rho_{+}(G)=\rho_{+}\left(F_{t_{0}}\right)$, i.e., $\rho_{+}(G)=b$.

Lemma 3.13. Let $P$ be a cycle such that every cycle with period $|P|$ and rotation number $\rho(P)$ which is forced by $P$ is equivalent to $P$. Then $\rho_{-}(P)=\rho_{+}(P)$. Proof. Let $P$ have period $n$ and rotation number $r / n$, and let $F$ be $P$ monotone.

Suppose $\rho_{-}(P) \neq \rho_{+}(P)$. Then by Lemma 3.10, $\rho_{-}(P)<r / n<\rho_{+}(P)$. Choose $a$ and $b$ so that $\rho_{-}(P)<a<r / n<b<\rho_{+}(P)$. By Lemma 3.12, there is a map $G$ which agrees with $F$ on its nonconstant part, such that $\rho_{-}(G)=a$ and $\rho_{+}(G)=b$. By Lemma 3.11, $G$ has a cycle $Q$, with period $n$ and rotation number $r / n$, which lies on the nonconstant part of $G$. Thus $Q$ is an $F$-cycle as well. But $\rho_{-}(P)<\rho_{-}(G) \leq \rho_{-}(Q)$. Therefore $P$ and $Q$ are not equivalent.

An immediate consequence of Lemma 3.13 is

Theorem 3.14. Primary cycles have trivial rotation intervals.

Proof of Theorem 3.3. Suppose every cycle of period $|P|$ and rotation number $\rho(P)$ which is forced by $P$ is equivalent to $P$. Let $P$ force $Q$, where $Q$ is of period $|P|$. It follows from Lemma 3.6 that $\rho_{-}(P) \leq \rho_{-}(Q) \leq \rho_{+}(Q) \leq \rho_{+}(P)$. By Lemma 3.13, $\rho_{-}(P)=\rho_{+}(P)$. Therefore $\rho(P)=\rho(Q)$, and hence $P$ and $Q$ are equivalent.

The converse of Theorem 3.14 is false. We construct an example of a cycle with a trivial rotation interval which is not primary.

Example. Let $\varphi: P \rightarrow P=\left\{\cdots<x_{0}<x_{1}<\cdots\right\}$ and $\psi: Q \rightarrow Q=\{\cdots<$ $\left.y_{0}<y_{1}<\cdots\right\}$ be cycles of period 6 and rotation number $\frac{1}{2}$, defined as follows. Let $\alpha(0,1,2,3,4,5)=(4,5,3,7,8,6)$, i.e., $\alpha(0)=4, \alpha(1)=5$, 
etc., and $\varphi\left(x_{6 i+k}\right)=x_{6 i+\alpha(k)}$. Let $\beta(0,1,2,3,4,5)=(3,5,4,8,7,6)$ and $\psi\left(y_{6 i+k}\right)=y_{6 i+\beta(k)}$. Then $P$ has a trivial rotation interval by Theorem 3.15 below. However, since $P$ forces $Q$ (as can be verified by examining the Markov graph of $P), P$ is not primary.

Definition. A cycle $\varphi: P \rightarrow P$ of period $n$ has a block structure over a cycle $\psi: Q \rightarrow Q=\left\{\cdots<y_{0}<y_{1}<\cdots\right\}$ of period $m$ if $n$ is a multiple of $m$, say $n=s m$, and $P$ can be written $P=\bigcup_{i \in \mathbf{Z}} P_{i}$, where $P_{i}=\left\{x_{i s}<\cdots<x_{(i+1) s-1}\right\}$, in such a way that $\varphi\left(P_{i}\right)=P_{j}$ if and only if $\psi\left(y_{i}\right)=y_{j}$.

Theorem 3.15. A cycle has a trivial rotation interval if and only if it has a block structure over a twist cycle.

Proof. Suppose $\varphi: P \rightarrow P$ has a trivial rotation interval, and let $F$ be $P$ monotone. By Lemma 3.8, $F$ has a twist cycle $Q$ such that $\rho(P)=\rho(Q)$, and for all $x \in P$ and $y \in Q, F(x)<F(y)$ if and only if $x<y$. Write $Q=\left\{\cdots<y_{0}<y_{1}<\cdots\right\}$ and let $\psi=\left.F\right|_{Q}$ and $P_{i}=P \cap\left[y_{i}, y_{i+1}\right)$. Then each block $P_{i}$ has the same number of points, and $\varphi\left(P_{i}\right)=P_{j}$ if and only if $\psi\left(y_{i}\right)=\psi\left(y_{j}\right)$. Thus $P$ has a block structure over $Q$.

Conversely, suppose $P$ has a block structure $P=\bigcup P_{i}$ over a twist cycle with rotation number $r / n$ (in lowest terms). Let $F$ be $P$-monotone. Then $F\left(P_{i}\right)=P_{i+r}$ and $F^{n}\left(P_{i}\right)=P_{i}+r$. Let $G_{i}=\left[\max P_{i}, \min P_{i+1}\right]$ be the gap between $P_{i}$ and $P_{i+1}$. Then $F\left(G_{i}\right) \supseteq G_{i+r}$ and $F^{n}\left(G_{i}\right) \supseteq G_{i}+r$. Hence, by the standard Markov graph argument, there exist $y_{i} \in G_{i}$ such that $F\left(y_{i}\right)=y_{i+r}$ and $F^{n}\left(y_{i}\right)=y_{i}+r$. Then $Q=\left\{\cdots<y_{0}<y_{1}<\cdots\right\}$ is a twist cycle, and $P, Q$, and $F$ satisfy the hypotheses of Lemma 3.9. Hence $P$ has a trivial rotation interval.

\section{A CHARACTERIZATION OF PRIMARY CYCLES}

Definition. Let $\varphi: P \rightarrow P$ be a cycle which has a block structure $P=\bigcup P_{i}$ over a cycle $Q$ of period $n$ and rotation number $r / n$, and let $X$ be an interval cycle. $P$ is an $X$-extension of $Q$ if for some $k, 0 \leq k \leq n-1, \varphi$ is monotone on $P_{i}$ except possibly when $i \equiv k(\bmod n)$, and $P_{k}$ is a $\left(\varphi^{n}-r\right)$-cycle equivalent to $X$. As in the interval case, a 2-extension is one in which $X$ is a two-point cycle, and a Štefan extension is one in which $X$ is a Štefan cycle.

If $P$ is an $X$-extension as above, then for $i \not \equiv k(\bmod n), P_{i}$ is a $\left(\varphi^{n}-r\right)$ cycle equivalent to either $X$ or its "mirror image"-obtained by conjugating $X$ by an orientation-reversing homeomorphism of the interval, i.e., looking at $X$ upside-down and backward.

Note that if $P$ has a block structure over $Q$ and $|P|=2|Q|$, then $P$ is a 2-extension of $Q$.

Example. The cycle $\psi: Q \rightarrow Q$ in the example at the end of $\S 3$ is a Stefan extension of a twist cycle of period 2 and rotation number $\frac{1}{2}$. 
Theorem 4.1. The following statements about a cycle $P$ are equivalent.

(1) $P$ is primary.

(2) Every cycle of period $|P|$ and rotation number $\rho(P)$ which is forced by $P$ is equivalent to $P$.

(3) $P$ can be obtained from a twist cycle by a finite number (possibly zero) of 2-extensions and then at most one Stefan extension.

(4) There is a map in $\mathscr{C}$ whose only cycle of period $|P|$ is $P$.

Note that $(4) \Rightarrow(2)$ is obvious and that $(2) \Rightarrow(1)$ is Theorem 3.3. Thus we need only prove $(3) \Rightarrow(4)$ and $(1) \Rightarrow(3)$.

Proof of Theorem 4.1. (3) $\Rightarrow(4)$. The proof is similar to that of Theorem 1.1, $(2) \Rightarrow(3)$, with the twist cycle playing the role of the one-point cycle. Let $\varphi: P \rightarrow P=\left\{\cdots<x_{0}<x_{1}<\cdots\right\}$.

If $P$ is a twist cycle, then (4) holds by Theorem 3.2. If $P$ is obtained from a twist cycle by taking a finite, nonzero number of 2-extensions, renumber $P$ so that $P=\bigcup P_{i}$, where $P_{i}=\left\{x_{i s}<\cdots<x_{(i+1) s-1}\right\}$, exhibits the block structure of $P$ over the twist cycle. In this case, let $F$ agree with $\varphi$ on $P$ and be monotone quadratic on $\left[x_{i}, x_{i+1}\right]$ for $i \equiv 0(\bmod |P|)$ and linear on $\left[x_{i}, x_{i+1}\right]$ for $i \not \equiv 0(\bmod |P|)$. If $P$ is obtained from a twist cycle by taking a finite number (possibly zero) of 2-extensions and then taking a Stefan extension, let $F$ agree with $\varphi$ on $P$ and be linear on each $\left[x_{i}, x_{i+1}\right]$. In the latter two cases, it is easy to see, using the Markov graph, that $P$ is the only $F$-cycle of period $|P|$.

It remains only to prove $(1) \Rightarrow(3)$.

Definition. For $A \subseteq \mathbf{R}$ closed and bounded, let $[A]=[\min A, \max A] \operatorname{denote}$ the convex hull of $A$.

Lemma 4.2. Let $Q$ be a cycle which has a block structure $Q=\bigcup Q_{i}$. Suppose $G$ is $Q$-monotone and has a cycle $S$ which has a block structure $S=\bigcup S_{i}$ such that $S_{i} \subseteq\left[Q_{i}\right]$ for each $i$. If $Q$ is a cycle of a map $F \in \mathscr{C}$, then $F$ has a cycle $R$ with a block structure $R=\bigcup R_{i}$ such that $|R|=|S|$ and $R_{i} \subseteq\left[Q_{i}\right]$ for each $i$.

Proof. This follows in the same way as does Lemma 2.2.

Lemma 4.3 (cf. [ALM, Lemma 3.13]). Let $\varphi: P \rightarrow P$ be a primary cycle which has a block structure $P=\bigcup P_{i}$ over a cycle of period $n$ and rotation number $r / n$. Then each block $P_{i}$ is a primary interval cycle of $\varphi^{n}-r$.

Proof. Suppose not. By renumbering the blocks, we may assume that $P_{0}$ is a $\left(\varphi^{n}-r\right)$-cycle which is not primary.

By Lemma 2.4 , there is a $P$-monotone map $F$ which has only finitely many cycles of each period. Since $F^{n}-r$ maps $\left[P_{0}\right]$ to itself, $F^{n}-r$ has a cycle $Q_{0}$ of period $\left|P_{0}\right|$ which is properly contained in $\left[P_{0}\right]$. We may choose $Q_{0}$ so that $\left[Q_{0}\right]$ contains no other $\left(F^{n}-r\right)$-cycle of period $\left|P_{0}\right|$. Then $Q=$ 
$\bigcup_{k \geq 0, s \in \mathbf{Z}}\left(F^{k}\left(Q_{0}\right)+s\right)$ is an $F$-cycle which has a block structure $Q=\bigcup Q_{i}$, with each $Q_{i} \subseteq\left[P_{i}\right]$, over the same cycle as does $P$. Since $|P|=|Q|, P$ and $Q$ are equivalent. The equivalence respects the block structures. Let $P_{0}$ correspond to $Q_{j}$.

Now apply the argument given above to $Q_{j}$. By Lemma 4.2, there is an $F$-cycle $R$ of period $|P|$, with a block structure $R=\bigcup R_{i}$ where each $R_{i}$ is an $\left(F^{n}-r\right)$-cycle of period $\left|P_{0}\right|$, properly contained in $\left[Q_{i}\right]$. This violates the choice of $Q_{0}$.

Lemma 4.4 (cf. [ALM, Lemma 8.2]). Let $P$ be primary and suppose $P$ has a block structure over $Q$. If $|P| /|Q|$ is even, then $P$ has a block structure over some 2-extension of $Q$.

Proof. Let $\varphi: P \rightarrow P=\bigcup P_{i}$ exhibit the block structure, and suppose $Q$ has period $n$ and rotation number $r / n$. Each block $P_{i}$ has $|P| /|Q|$ points.

It suffices to show that $\varphi\left(\left\{L_{i}, R_{i}\right\}\right)=\left\{L_{j}, R_{j}\right\}$ whenever $\varphi\left(P_{i}\right)=P_{j}$, where $L_{i}$ and $R_{i}$ are the left and right halves of $P_{i}$.

Fix $i$. By Lemma 4.3, $P_{i}$ is a primary interval cycle of $\varphi^{n}-r$. By Theorem $1.1,(1) \Rightarrow(2), \varphi^{n}-r$ interchanges $L_{i}$ and $R_{i}$, and so for every even integer $k>0, \varphi^{k n}-k r$ maps $L_{i}$ to $L_{i}$ and $R_{i}$ to $R_{i}$. Now suppose $\varphi\left(P_{i}\right)=P_{j}$ and $x$ and $y$ both lie in $L_{i}$ or both lie in $R_{i}$. There is an even integer $k>0$ such that $y=\varphi^{k n}(x)-k r$. If $\varphi(x) \in L_{j}$, then so is $\varphi^{k n}(\varphi(x))-k r=\varphi(y)$. Similarly, if $\varphi(x) \in R_{j}$, then so is $\varphi(y)$. Thus $\varphi\left(\left\{L_{i}, R_{i}\right\}\right)=\left\{L_{j}, R_{j}\right\}$.

Lemma 4.5. Let $f$ be an interval map which has a cycle $X$ of period sm, where $m \geq 3$ is odd. If $X$ has a block structure $X=X_{1} \cup \cdots \cup X_{\text {s }}$ over some cycle of period $s$, then $f$ has a cycle $Y$, also of period sm, which has a block structure $Y=Y_{1} \cup \cdots \cup Y_{s}$ over the same cycle as does $X$, such that each $Y_{i}$ is a Stefan cycle of $f^{s}$ of period $m$, contained in the convex hull of $X_{i}$, and except for exactly one $i, f$ maps $Y_{i}$ monotonically onto $f\left(Y_{i}\right)$.

Proof. This result is proved in [BC] under the assumption that $s$ is a power of two. An examination of the proof reveals that this assumption is unnecessary.

Proof of Theorem 4.1. (1) $\Rightarrow(3)$. Let $P$ be primary. By Theorems 3.14 and $3.15, P$ has a block structure over a twist cycle. Applying Lemma 4.4 the appropriate number of times, we find that $P$ has a block structure $P=\bigcup P_{i}$ over a cycle $Q$ which is obtained from the twist cycle by taking a finite number (possibly zero) of 2-extensions, and such that $|P| /|Q|$ is odd.

If $|P| /|Q|=1$, then $P$ is equivalent to $Q$ and we are done. So suppose that $|P| /|Q|=m$, where $m \geq 3$ is odd. Let $F$ be $P$-monotone and suppose that $Q$ has period $s$ and rotation number $t / s$. Let $X=P_{0} \cup F\left(P_{0}\right) \cup \cdots \cup F^{s-1}\left(P_{0}\right)$, and define $f:[X] \rightarrow[X]$ as follows. Let $f$ agree with $F$ on $\left[P_{0}\right] \cup \cdots \cup F^{s-2}\left(\left[P_{0}\right]\right)$, $f(x)=F(x)-t$ on $F^{s-1}\left(\left[P_{0}\right]\right)$, and then extend $f$ to an interval map of $[X]$ to itself which is linear between $F^{i}\left(\left[P_{0}\right]\right)$ and $F^{j}\left(\left[P_{0}\right]\right)$ whenever these intervals 
are "adjacent." Then $f$ and $X$ satisfy the hypotheses of Lemma 4.5. Let $Y$ be an $f$-cycle whose existence is guaranteed by Lemma 4.5. Then $R=\bigcup_{k \in Z}(Y+k)$ is an $F$-cycle which is a Stefan extension of $Q$. By Lemma 2.2, $P$ forces $R$. But $P$ is primary, so $P$ is equivalent to $R$. Thus $P$ is a Stefan extension of $Q$.

An alternate proof of Theorem $4.1,(1) \Rightarrow(3)$ may be obtained by adapting the lemmas of $[A L M, \S 8]$ to the circle.

\section{REFERENCES}

[ALM] L. Alsedà, J. Llibre and M. Misiurewicz, Periodic orbits of maps of $Y$, preprint, Barcelona, 1987.

[ALMS] L. Alsedà, J. Llibre, M. Misiurewicz and C. Simó, Twist periodic orbits and topological entropy for continuous maps of the circle of degree one which have a fixed point, Ergodic Theory Dynamical Systems 5 (1985), 501-517.

[ALS] L. Alsedà, J. Llibre and R. Serra, Minimal periodic orbits for maps of the interval, Trans. Amer. Math. Soc. 286 (1984), 595-627.

[Ba] S. Baldwin, Generalizations of a theorem of Šarkovskii on orbits of continuous real-valued functions, Discrete Math. 67 (1987), 111-127.

[Be] C. Bernhardt, Simple permutations with order a power of two, Ergodic Theory Dynamical Systems 4 (1984), 179-186.

[Bl] L. Block, Simple periodic orbits of mappings of the interval, Trans. Amer. Math. Soc. 254 (1979), 391-398.

[BC] L. Block and W. A. Coppel, Stratification of continuous maps of an interval, Trans. Amer. Math. Soc. 297 (1986), 587-604.

[CGT] A. Chenciner, J.-M. Gambaudo and Ch. Tresser, Une remarque sur la structure des endomorphismes de degré 1 du cercle, C. R. Acad. Sci. Paris 299 (1984), A145-A148.

[C] W. A. Coppel, Šarkovskii-minimal orbits, Math. Proc. Cambridge Philos. Soc. 93 (1983), $397-$ 408.

[H] C.-W. Ho, On the structure of minimum orbits of periodic points for maps of the real line, preprint, Southern Illinois Univ., Edwardsville, 1982.

[L] J. Llibre, Minimal periodic orbits of continuous mappings of the circle, Proc. Amer. Math. Soc. 83 (1981), 625-628.

[MT] R. MacKay and Ch. Tresser, Badly ordered orbits of circle maps, Math. Proc. Cambridge Philos. Soc. 96 (1984), 447-451.

[M1] M. Misiurewicz, Periodic points of maps of degree one of a circle, Ergodic Theory Dynamical Systems 2 (1982), 221-228.

[M2] __, Twist sets for maps of the circle, Ergodic Theory Dynamical Systems 4 (1984), 391-404.

[M3] _ _ Rotation intervals for a class of maps of the real line into itself, Ergodic Theory Dynamical Systems 6 (1986), 117-132.

[NPT] S. Newhouse, J. Palis and F. Takens, Bifurcations and stability for families of diffeomorphisms, Inst. Hautes Ètudes Sci. Publ. Math. 57 (1983), 5-72.

[Š] P. Štefan, A theorem of Šarkovskii on the existence of periodic orbits of continuous endomorphisms of the real line, Comm. Math. Phys. 54 (1977), 237-248.

Department of Mathematics, University of Florida, Gainesville, Florida 32611

Department of Mathematics, Wesleyan University, Middletown, Connecticut 06457

Department of Mathematics and Statistics, Queen's University, Kingston, Ontario K7L 3N6 CANADA

Instytut Matematyki, Uniwersytet Warszawski, 00-901 Warszawa, Poland 